\title{
STRONG BOUNDEDNESS OF ANALYTIC FUNCTIONS IN TUBES
}

\author{
RICHARD D. CARMICHAEL \\ Department of Mathematics \\ Iowa State University \\ Ames, Iowa 50011 \\ U.S.A. \\ (Received October 30, 1978)
}

ABSTRACT. Certain classes of analytic functions in tube domains $T^{C}=\mathbb{R}^{n}+i C$ in $n$-dimensional complex space, where $C$ is an open connected cone in $\mathbb{R}^{n}$, are studied. We show that the functions have a boundedness property in the strong topology of the space of tempered distributions $8^{\prime}$. We further give a direct proof that each analytic function attains the Fourier transform of its spectral function as distributional boundary value in the strong (and weak) topology of $8^{\prime}$.

KEY WORDS AND PHRASES. Analytic Function in Tubes, Strong Boundedness, Tempered Distributions, Distributional Boundary Value.

AMS (MOS) SUBJECT CLASSIFICATION (1970) CODES. 32A07, 32A10, $46 F 10$.

1. INTRODUCTION.

Vladimirov [1, p. 230] has defined the spectral function $v_{t}$ of a function $f(z)$ which is analytic in a tubular domain $T^{B}=\mathbb{R}^{n}+i B$ to be the distribution 
$v_{t} \varepsilon D^{\prime}$, the space of distributions of L. Schwartz [2], which possesses the following properties:

$$
\begin{gathered}
e^{-y t} v_{t} \varepsilon 8^{\prime} \quad \text { for all y } \varepsilon B ; \\
f(z)=\left\langle v_{t}, e^{i z t}\right\rangle \text { for all } z \varepsilon T^{B} .
\end{gathered}
$$

Here $\delta^{\prime}$ is the space of tempered distributions of Schwartz [2] and $\left\langle v_{t}, e^{i z t}\right\rangle$ is the Fourier-Laplace transform of the spectral function $v_{t}$.

In [3] Vladimirov defined certain classes of analytic functions in tubular cones $T^{C}=\mathbb{R}^{n}+1 C$, where $C$ is an open cone, and analyzed the spectral functions of these analytic functions corresponding to $\mathrm{C}$ being an open connected cone. The results of [3] have been incorporated into the book [1] of vladimirov $[1$, section 26.4$]$.

In this paper we add information to the main results of [3] and [1, section 26.4] which are [1, pp. 238-239, Theorems 1 and 2]. We show that the analytic functions considered by vladimirov in these results have boundedness properties In the strong topology of the space of tempered distributions $8^{\prime}$. Further, we give a direct proof by elementary means that each analytic function attains the Fourier transform of its spectral function as distributional boundary value in the strong (and weak) topology of $\mathbf{8}^{\prime}$, a fact which has been recognized by Vladimirov [1, p. 238] and which is obtained by him as a special case of a more general result.

\section{NOTATION AND DEFINITIONS.}

Our n-dimensional notation is that of vladimirov $[1, p, 1] . x, y$, and $t$ will be points in $\mathbb{R}^{\mathrm{n}}$ in this paper and $z \in \mathbb{C}^{\mathrm{n}}, \mathrm{n}$-dimensional complex space. Note the inner products $z t=z_{1} t_{1}+\ldots+z_{n} t_{n}$ and $y t=y_{1} t_{1}+\ldots+y_{n} t_{n}$ for $t$ and $y$ in $\mathbb{R}^{\mathrm{n}}$ and $z \in \mathbb{C}^{\mathrm{n}}$. Note also the differential operator $D^{\alpha}$ in $[1, \mathrm{p} .1]$, and we shall write $D_{z}^{\alpha}$ or $D_{t}^{\alpha}$ to indicate that the differentiation is with respect to $z$ or $t$, respectively. Here $\alpha$ is an $n$-tuple of nonnegative integers. The 
definitions of cone $C$ in $\mathbf{R}^{n}$, compact subcone of a cone, indicatrix $u_{C}(t)$ of a cone, and of the number $\rho_{C}$, which characterizes the nonconvexity of a cone $C$, can all be found in [1, section 25.1]. Note that $\rho_{C} \geq 1$ [1, p. 220] for any cone $C$. The cone $C^{*}=\left\{t \varepsilon \mathbb{R}^{n}: y t \geq 0, y \varepsilon C\right\}$ is the dual cone of $C$ and $C_{*}$ will denote $C_{*}=\mathbb{R}^{n} \backslash C^{*} \cdot O(C)$ will denote the convex envelope (hull) of the cone $C$, and we define the tubes $T^{C}$ and $T^{0(C)}$ by $T^{C}=\mathbb{R}^{n}+i C$ and $T^{0(C)}=\mathbf{R}^{n}+i 0(C)$, respectively.

Let $C$ be a cone in $\mathbb{R}^{n}$. We make the convention throughout this paper that by $z \in \mathrm{T}^{\mathrm{C}}\left(\varepsilon \mathrm{T}^{\mathrm{O}(\mathrm{C})}\right)$ and $\mathrm{y} \varepsilon \mathrm{C}(\varepsilon 0(\mathrm{C}))$ we mean that $\mathrm{z} \varepsilon \mathrm{T}^{C^{\prime}}$ and y $\varepsilon C^{\prime}$ for an arbitrary compact subcone $C^{\prime} \subset C \quad\left(C^{\prime} \subset 0(C)\right)$.

The space of functions of rapid decrease $\delta=8\left(\mathbb{R}^{n}\right)$ and the space of tempered distributions $\delta^{\prime}=g^{\prime}\left(\mathbb{R}^{n}\right)$ are defined and discussed in Schwartz [2, Chapter 7]. The Fourier (inverse Fourier) transform of an $L^{1}\left(\mathbb{R}^{n}\right)$ function $\phi(t)$, denoted $₹[\phi(t) ; x]\left(\mathcal{F}^{-1}[\phi(t) ; x]\right)$, will be as defined in vladimirov $\left[1\right.$, p. 21]. The Fourier transform of a tempered distribution $v_{t}$, denoted J[V], is defined in Schwartz [2, p. 250, (VII 6;6)]. A11 terminology and definitions concerning distributions in this paper, such as support of a distribution, wil1 be that of Schwartz [2].

Let $C$ be an open connected cone. The analytic function $f(z), z \varepsilon T^{C}$, obtains $U \in 8^{\prime}$ as boundary value in the weak topology of $8^{\prime}$ if

$$
\lim _{y \rightarrow 0}\langle f(x+i y), \phi(x)\rangle=\langle U, \phi\rangle
$$

for each $\phi \varepsilon \&$. $U \varepsilon \mathrm{g}^{\prime}$ is the boundary value of $f(z)$ in the strong topology of $\delta^{\prime}$ if the convergence (2.1) holds uniformly for $\phi$ varying over arbitrary bounded sets in 8 . The set $\left\{U_{y} \varepsilon \mathcal{S}^{\prime}: y \varepsilon C\right\}$, where $U_{y} \varepsilon g^{\prime}$ in some sense depends on $y \varepsilon C$, is said to be a bounded set in the strong topology of $g^{\prime}$ if for any bounded set $\Phi$ in $8,\left\{\left\langle U_{y}, \phi\right\rangle: \phi \varepsilon \Phi, y \varepsilon C\right\}$ is a bounded set in the complex plane. 


\section{THE THEOREMS OF VLADIMIROV.}

Let $C$ be an open cone. A function $f(z)$ belongs to the class $H_{p}(a ; C)$, where $p \geq 1$ and $a \geq 0$, if $f(z)$ is analytic in the tubular cone $T^{C}$ and, for an arbitrary compact subcone $C^{\prime}$ in $C$, the inequality

$$
|f(z)| \leq M\left(C^{\prime}\right)(1+|z|)^{N}\left(1+|y|^{-K}\right) e^{a|y|^{p}}, z=x+1 y \varepsilon T^{C^{\prime}},
$$

is satisfied where $M\left(C^{\prime}\right)$ is a constant which depends at most on the compact subcone $C^{\prime} \subset C$ and $N$ and $K$ are nonnegative real numbers which do not depend on $C^{\prime} \subset C$. We define

$$
H_{p}(a+\epsilon ; C)=a^{\prime} \bigcap_{a} H_{p}\left(a^{\prime} ; C\right), H_{0}(C)=H_{1}(0 ; C) .
$$

For the convenience of the reader we now state the theorems of vladimirov with which we are concerned in this paper.

THEOREM 1. [1, p. 238] Let $f(z) \in H_{p}(a+\epsilon ; C)$, where $C$ is an open connected cone, $p>1$, and $a>0$. The spectral function $v_{t}$ of $f(z)$ can be represented in the form of a finite sum of distributional derivatives of continuous functions $g_{\alpha}(t)$ of power increase,

$$
v_{t}=\sum_{\alpha} D_{t}^{\alpha}\left(g_{\alpha}(t)\right)
$$

which, for all $t \in C_{*}^{\prime}$, where $C_{*}^{\prime}$ is an arbitrary compact subcone of $C_{*}=\mathbb{R}^{n} \backslash C^{*}$, and for all $\epsilon>0$, satisfy

$$
\left|g_{\alpha}(t)\right| \leq M_{\epsilon}^{\prime}\left(C_{*}^{\prime}\right) \quad \exp \left[-\left(a^{\prime}-\epsilon\right)\left(u_{C}(t)\right)^{p^{\prime}}\right]
$$

where the numbers $\mathrm{p}$ and $\mathrm{a}$ are connected with $\mathrm{p}^{\prime}$ and $\mathrm{a}^{\prime}$ by the relations

$$
\frac{1}{p}+\frac{1}{p^{\prime}}=1,\left(p^{\prime} a^{\prime}\right)^{p}(p a)^{p^{\prime}}=1 \text {. }
$$

Conversely, if $v_{t}$ satisfies these conditions for certain numbers $a^{\prime}>0$, $p^{\prime}>1$ and the cone $C_{*}$, then all derivatives $D_{z}^{\beta}(f(z))$ of its FourierLaplace transform $f(z)$ belong to the class $H_{p}\left(a \rho_{C}^{p}+\epsilon ; 0(C)\right)$.

Notice that the $C^{*}$ as printed in $\left[1\right.$, p. 239, line 8] should be $C_{*}$ instead as we have written in Theorem 1 . 
THEOREM 2. [1, p. 239] Let $f(z) \varepsilon H_{1}(a+\epsilon: C)$ where $C$ is an open connected cone and $a \geq 0$. Then its spectral function $v_{t} \varepsilon g^{\prime}$ and $v_{t}$ has support in $\left\{t: u_{C}(t) \leq a\right\}$. Conversely, if $v_{t} \varepsilon g^{\prime}$ and has support in $\left\{t: u_{C}(t) \leq a\right\}$ for some $a \geq 0$ and some open connected cone $C$, then all the derivatives $D_{z}^{\beta}(f(z))$ of the Fourier-Laplace transform $f(z)$ of $v_{t}$ belong to the class $\mathrm{H}_{1}\left(\mathrm{a} \rho_{\mathrm{C}} ; \mathrm{O}(\mathrm{C})\right)$.

4. LEMMAS.

As noted in the introduction, we shall add information to Theorems 1 and 2 . We sha11 show that the analytic functions in these theorems have a strong boundedness property in $8^{\prime}$. In addition we give a direct proof that the analytic functions attain the Fourier transform of their spectral functions as distributional boundary values in the strong (and weak) topology of $8^{\prime}$.

The following lemma is the basis of the boundary value result, and its proof in turn is useful in obtaining our strong boundedness properties. Throughout this section $C$ is an open connected cone.

LEMMA 1. Let $f(z) \varepsilon H_{p}(a+\epsilon ; C), p>1$ and $a>0$. The spectral function $v_{t}$ of $f(z)$ is in $g^{\prime}$ as is $\left(e^{-y t} v_{t}\right), y \varepsilon 0(C)$, and

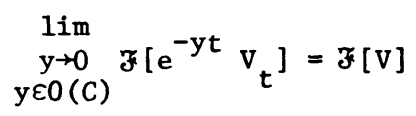

in the strong (and weak) topology of $\mathbf{8}^{\prime}$.

PROOF. Let $C^{\prime}$ be an arbitrary compact subcone of $0(C)$. By the sufficiency of Theorem 1 , the spectral function $v_{t}$ of $f(z)$ has the representation (3.2). Since each $g_{\alpha}(t)$ in (3.2) is continuous and of power increase over $\mathbb{R}^{n}$, we immediately have $v_{t} \varepsilon 8^{\prime}$. The fact that $\left(e^{-y t} v_{t}\right) \varepsilon 8^{\prime}$, y $\varepsilon C^{\prime} \subset 0(C)$, follows by the proof of Theorem 1 given in [1, section 26.5]. Let $\phi$ be an arbitrary element of 8 . Using the notion of distributional differentiation and the generalized Leibnitz rule, we have for $y \varepsilon C^{\prime} \subset 0(C)$ that 


$$
\begin{aligned}
& \left\langle V_{t},\left(e^{-y t}-1\right) \phi(t)\right\rangle= \\
= & \sum_{\alpha}(-1)^{|\alpha|} \int_{\mathbb{R}^{n}} g_{\alpha}(t) \sum_{\beta+\gamma=\alpha} \frac{\alpha !}{\beta ! \gamma !} D_{t}^{\beta}\left(e^{-y t}-1\right) D_{t}^{\gamma}(\phi(t)) d t \\
= & \sum_{\alpha}(-1)^{|\alpha|} \sum_{\beta+\gamma=\alpha} \frac{\alpha !}{\beta ! \gamma !} I_{y}(\alpha, \beta, \gamma)
\end{aligned}
$$

where $\alpha, \beta$, and $\gamma$ are n-tuples of nonnegative integers and

$$
I_{y}(\alpha, \beta, \gamma)=\int_{\mathbb{R}^{n}} g_{\alpha}(t)\left((-1)^{|\beta|} y^{\beta} e^{-y t}-D_{t}^{\beta}(1)\right) D_{t}^{\gamma}(\phi(t)) d t .
$$

For the arbitrary $C^{\prime} \subset O(C)$ we apply [1, p. 223, Lemma 2] to obtain a number $\delta=\delta\left(C^{\prime}\right)>0$ and an open cone $\left(C^{*}\right)^{\prime}$, both depending on $C^{\prime}$, such that $\left(C^{*}\right)^{\prime}$ contains the cone $C^{*}=\left\{t \in \mathbb{R}^{n}:\right.$ yt $\left.\geq 0, y \in C\right\}$, the dual cone of $c$, and

$$
\text { yt } \geq \delta|y||t|, \quad y \varepsilon c^{\prime}, \quad t \varepsilon\left(C^{*}\right)^{\prime} \text {. }
$$

Put $C_{*}^{\prime}=\mathbb{R}^{n} \backslash\left(C^{*}\right)^{\prime}$. $C_{*}^{\prime}$ is a compact subcone of $C_{*}=\mathbb{R}^{n} \backslash C^{*}$, and we have $c_{*}^{\prime} \cap\left(C^{*}\right)^{\prime}=\varnothing$ and $C_{*}^{\prime} \cup\left(C^{*}\right)^{\prime}=\mathbb{R}^{n}$. We now write the integral $I_{y}(\alpha, \beta, \gamma)$ in $(4.3)$ as

$$
I_{y}(\alpha, \beta, \gamma)=I_{y}^{1}(\alpha, \beta, \gamma)+I_{y}^{2}(\alpha, \beta, \gamma)
$$

where

$$
\begin{aligned}
& I_{y}^{1}(\alpha, \beta, \gamma)=\int_{\left(C^{*}\right)} g_{\alpha}(t)\left((-1)|\beta| y^{\beta} e^{-y t}-D_{t}^{\beta}(1)\right) D_{t}^{\gamma}(\phi(t)) d t \\
& I_{y}^{2}(\alpha, \beta, \gamma)=\int_{C_{*}} g_{\alpha}(t)\left((-1)^{|\beta|} y^{\beta} e^{-y t}-D_{t}^{\beta}(1)\right) D_{t}^{\gamma}(\phi(t)) d t .
\end{aligned}
$$

For any n-tuple $\beta$ of nonnegative integers we have

$$
(-1)^{|\beta|} y^{\beta} e^{-y t}-D_{t}^{\beta}(1)=\left\{\begin{array}{l}
e^{-y t}-1, \beta=(0, \ldots, 0), \\
(-1)^{|\beta|} y^{\beta} e^{-y t}, \beta \neq(0, \ldots, 0),
\end{array}\right.
$$

for all y $\varepsilon C^{\prime} \subset 0$ (C) and in fact for all y $\varepsilon \mathbb{R}^{n}$; hence for any $\alpha$ in the last sum in (4.2) and any subsequent $\beta$ and $\gamma, \beta+\gamma=\alpha$, (4.7) yields 


$$
\lim _{\substack{y \rightarrow 0 \\ y \in 0(C)}} g_{\alpha}(t)\left((-1)|\beta| y^{\beta} e^{-y t}-D_{t}^{\beta}(1)\right) D_{t}^{Y}(\phi(t))=0
$$

for all $t \in \mathbb{R}^{\mathrm{n}}$. (The limit (4.8) actually holds as $\mathrm{y} \rightarrow 0$, $\mathrm{y} \varepsilon \mathbb{R}^{\mathrm{n}}$, because (4.7) holds for all y $\varepsilon \mathbb{R}^{\mathrm{n}}$.)

Recall that we desire a convergence result in this lemma as $y \rightarrow 0$, y $\varepsilon O(C)$. Hence to obtain (4.1) it suffices to consider y $\varepsilon O(C)$ such that $|y| \leq Q$ for $Q>0$ fixed. Now consider the integrand of the integral $\mathrm{I}_{\mathrm{y}}^{1}(\alpha, \beta, \gamma)$ in $(4.6)$ for $t \varepsilon\left(C^{*}\right)^{\prime}$. Since each $\mathrm{g}_{\alpha}(t)$ in $(3.2)$ is of power increase over $\mathbb{R}^{n}$, we have the existence of a polynomial $P_{\alpha}(t)$ corresponding to each $g_{\alpha}(t)$ such that

$$
\left|g_{\alpha}(t)\right| \leq P_{\alpha}(|t|), t \varepsilon \mathbb{R}^{n} \text {. }
$$

Using (4.9) and (4.4) we get

$$
\begin{aligned}
& \left|g_{\alpha}(t)\left((-1)^{|\beta|} y^{\beta} e^{-y t}-D_{t}^{\beta}(1)\right) D_{t}^{\gamma}(\phi(t))\right| \leq \\
& \leq P_{\alpha}(|t|)(1+|y||\beta| \exp (-\delta|y||t|))\left|D_{t}^{\gamma}(\phi(t))\right| \\
& \leq P_{\alpha}(|t|)\left(1+Q^{|\beta|}\right)\left|D_{t}^{\gamma}(\phi(t))\right|
\end{aligned}
$$

for $t \in\left(C^{*}\right)^{\prime}$ and $y \varepsilon C^{\prime} \subset O(C)$ such that $|y| \leq Q$. Since $\phi \varepsilon 8$, the right side of the last inequality in (4.10) is an $L^{1}$ function over $\mathbb{R}^{n}$ which is independent of $y \in C^{\prime} \subset O(C)$ such that $|y| \leq Q$. Using this fact, (4.8), and the Lebesgue dominated convergence theorem we obtain

$$
\lim _{y \rightarrow 0} \quad I_{y}^{1}(\alpha, \beta, \gamma)=0
$$

for any $\alpha$ in (4.2) and any subsequent $\beta$ and $\gamma, \beta+\gamma=\alpha$.

We now consider the integrand of the integral $\mathrm{I}_{\mathrm{y}}^{2}(\alpha, \beta, \gamma)$ in (4.6) for $t \varepsilon C_{*}^{\prime}$. For such $t$ each $g_{\alpha}(t)$ in (3.2) satisfies (3.3). Using (3.3), the relations $(3.4)$, the facts

$$
-y t \leq|y| u_{0(C)}(t), u_{0(C)}(t) \leq \rho_{C} u_{C}(t), \quad t \varepsilon c_{*}, \quad y \varepsilon 0(C)
$$


contained in [1, section 25.1], and analysis as in [1, p. 244], we have for $t \in C_{*}^{\prime} \subset C_{*}$ and $y \in C^{\prime} \subset O(C)$ such that $|y| \leq Q$ that

$$
\begin{aligned}
& \left|g_{\alpha}(t)\left((-1)|\beta| y^{\beta} e^{-y t}-D_{t}^{\beta}(1)\right) D_{t}^{\gamma}(\phi(t))\right| \leq \\
\leq & M_{\epsilon}^{\prime}\left(C_{*}^{\prime}\right) \exp \left[-\left(a^{\prime}-\epsilon\right)\left(u_{C}(t)\right)^{p^{\prime}}\right]\left(1+|y||\beta| e^{-y t}\right)\left|D_{t}^{\gamma}(\phi(t))\right| \\
\leq & M_{\epsilon}^{\prime}\left(C_{*}^{\prime}\right) \exp \left[-\left(a^{\prime}-\epsilon\right)\left(u_{C}(t)\right)^{p^{\prime}}\right]\left(1+|y|{ }^{|\beta|} \exp \left[|y| \rho_{C} u_{C}(t)\right]\right)\left|D_{t}^{\gamma}(\phi(t))\right| \\
\leq & M_{\epsilon}^{\prime}\left(C_{*}^{\prime}\right)(1+|y||\beta|) \exp \left[-\left(a^{\prime}-\epsilon\right)\left(u_{C}(t)\right)^{p^{\prime}}+|y| \rho_{C} u_{C}(t)\right]\left|D_{t}^{\gamma}(\phi(t))\right| \\
\leq & M_{\epsilon}^{\prime}\left(C_{*}^{\prime}\right)\left(1+Q^{|\beta|}\right) \exp \left[\frac{1}{p}\left(\frac{1}{p^{\prime}\left(a^{\prime}-2 \epsilon\right)}\right)^{p / p^{\prime}} \rho_{C}^{p}|y|^{p}\right]\left|D_{t}^{\gamma}(\phi(t))\right| .
\end{aligned}
$$

(3.3) holds for all $\epsilon>0$. In particular (3.3), and hence (4.13), holds for $\epsilon>0$ fixed such that $\left(a^{\prime}-2 \epsilon\right)>0$ for the fixed $a^{\prime}$ in (3.4). For $\epsilon>0$ fixed in this way in obtaining (4.13), we now conclude from (4.13) that

$$
\begin{gathered}
\left|g_{\alpha}(t)\left((-1){ }^{|\beta|} y^{\beta} e^{-y t}-D_{t}^{\beta}(1)\right) D_{t}^{\gamma}(\phi(t))\right| \leq \\
\leq M_{\epsilon^{\prime}}^{\prime}\left(C_{*}^{\prime}\right)\left(1+Q^{|\beta|}\right) \exp \left[\frac{1}{p}\left(\frac{1}{p^{\prime}\left(a^{\prime}-2 \epsilon\right)}\right)^{p / p^{\prime}} \rho_{C}^{p} Q^{p}\right]\left|D_{t}^{\gamma}(\phi(t))\right|
\end{gathered}
$$

for all $t \in C_{*}^{\prime} \subset C_{*}$ and $y \in C^{\prime} \subset 0(C)$ such that $|y| \leq Q$. Since $\phi \varepsilon \&$ the right side of (4.14) is an $L^{1}$ function over $\mathbb{R}^{\mathrm{n}}$ and is independent of y $\varepsilon C^{\prime} \subset O(C)$ such that $|y| \leq Q$. Thus by (4.14), (4.8), and the Lebesgue dominated convergence theorem we have

$$
\lim _{\substack{y \in 0 \\ y \in O(C)}} I_{y}^{2}(\alpha, \beta, \gamma)=0
$$

for each relevant $\alpha, \beta$, and $\gamma$. Combining (4.5), (4.11), and (4.15) we get

$$
\lim _{y \in 0} I_{y}(\alpha, \beta, \gamma)=0
$$

for each $\alpha$ in (4.2) and each $\beta$ and $\gamma, \beta+\gamma=\alpha$. Since $\phi$ is an arbitrary element of 8 , we combine $(4.2)$ and (4.16) to yield 


$$
\underset{y \in 0(C)}{\lim _{y \rightarrow 0}} e^{-y t} v_{t}=v_{t}
$$

In the weak topology of $g^{\prime}$. But $\delta$ is a Montel space $([1, p .21]$ and $[4$, p. 510].) Hence by Edwards [4, p. 510, Corollary 8.4.9] the convergence (4.17) is in the strong topology of $g^{\prime}$ also. Since the Fourier transform on $8^{\prime}$ [2, Chapter 7] is a strong1y continuous mapping of $8^{\prime}$ onto $\delta^{\prime}$, the desired convergence (4.1) now follows in the strong (and weak) topology of $8^{\prime}$. The proof is complete.

The next lemma is the basis of our strong boundedness results concerning the analytic functions $H_{p}(a+\epsilon ; C), p>1$ and $a>0$.

LEMMA 2. Let $\mathrm{p}>1$ and $\mathrm{a}>0$. Let $\mathrm{C}$ be an open connected cone. Let $v_{t}$ be any generalized function of the form (3.2) where the $g_{\alpha}(t)$ satisfy the conditions stated in Theorem 1. Then $v_{t} \varepsilon 8^{\prime},\left(e^{-y t} v_{t}\right) \varepsilon 8^{\prime}$ for all $\mathrm{y} \varepsilon O(\mathrm{C})$, and $\left\{z\left[\mathrm{e}^{-\mathrm{yt}} \mathrm{v}_{\mathrm{t}}\right] \varepsilon \mathrm{s}^{\prime}: \mathrm{y} \varepsilon 0(\mathrm{C}),|\mathrm{y}| \leq \mathrm{Q}\right\}$ is a strongly bounded set in $\delta^{\prime}$ for $Q>0$ being arbitrary but fixed.

PROOF. Let $C^{\prime}$ be an arbitrary compact subcone of $O(C)$. The facts that $v_{t} \varepsilon 8^{\prime}$ and $\left(e^{-y t} v_{t}\right) \varepsilon 8^{\prime}$ for all y $\varepsilon c^{\prime} \subset 0(C)$ follow as at the beginning of the proof of Lemma 1. The locally convex topology of 8 is defined by the norms

$$
\|\phi\|_{k}=\sup _{t \alpha \mid \leq k}(1+|t|)^{k}\left|D_{t}^{\alpha}(\phi(t))\right|, \quad k=1,2,3, \ldots
$$

Let $\Phi$ be an arbitrary bounded set in 8 . For the arbitrary $c^{\prime} \subset 0$ (C) we apply [1, p. 223, Lemma 2] as in the proof of Lemma 1 and obtain a number $\delta=\delta\left(C^{\prime}\right)>0$ and an open cone $\left(C^{*}\right)^{\prime}$, both depending on $C^{\prime}$, such that $\left(C^{*}\right)^{\prime}$ contains the cone $C^{*}$ and $(4.4)$ holds. We then put $C_{*}^{\prime}=\mathbf{R}^{n} \backslash\left(C^{*}\right)^{\prime}$, and $C_{*}^{\prime}$ is a compact subcone of $C_{*}=\mathbb{R}^{n} \backslash C^{*}$ as in the proof of Lemma 1 . Using the form of $v_{t}$ in (3.2) and the generalized Leibnitz rule we obtain for any $\phi \varepsilon \Phi$ and $\mathrm{y} \in \mathrm{C}^{\prime} \subset O(\mathrm{C})$ that 


$$
\left\langle e^{-y t} v_{t}, \phi(t)\right\rangle=\sum_{\alpha}(-1)|\alpha| \sum_{\beta+\gamma=\alpha} \frac{\alpha !}{\beta ! \gamma !}(-1)^{|\beta|} y^{\beta}\left(I_{y}^{1}(\alpha, \gamma)+I_{y}^{2}(\alpha, \gamma)\right)
$$

where

$$
\begin{aligned}
& I_{y}^{1}(\alpha, \gamma)=\int_{\left(C^{*}\right)}, g_{\alpha}(t) e^{-y t} D_{t}^{\gamma}(\phi(t)) d t \\
& I_{y}^{2}(\alpha, \gamma)=\int_{C_{*}^{1}} g_{\alpha}(t) e^{-y t} D_{t}^{\gamma}(\phi(t)) d t .
\end{aligned}
$$

Using (4.4), (4.18), and the fact that each $g_{\alpha}(t)$ satisfies (4.9) for some polynomial $P_{\alpha}(t)$, we have

$$
\begin{aligned}
& \left|I_{y}^{1}(\alpha, \gamma)\right| \leq \int_{\left(C^{*}\right)}, P_{\alpha}(|t|) \exp [-\delta|y||t|]\left|D_{t}^{\gamma}(\phi(t))\right| d t \\
& \quad \leq \int_{\left(C^{*}\right)}, P_{\alpha}(|t|)(1+|t|)^{n+1}\left|D_{t}^{\gamma}(\phi(t))\right|(1+|t|)^{-n-1} d t \\
& \quad \leq R_{\alpha}\|\phi\|_{k_{\alpha}} \int_{\mathbb{R}^{n}}(1+|t|)^{-n-1} d t
\end{aligned}
$$

where $R_{\alpha}$ is a constant and $k_{\alpha}$ is a positive integer with both depending on $\alpha$; and (4.21) holds for each $\alpha$ and $\gamma, \alpha=\beta+\gamma$, in (4.19). Also recall that each $g_{\alpha}(t)$ satisfies (3.3). Using (3.3), (4.12), and analysis as in (4.21), (4.13), and (4.14) we have for $y \in C^{\prime} \subset 0(C)$ that

$$
\begin{aligned}
& \left|I_{y}^{2}(\alpha, \gamma)\right| \leq M_{\epsilon^{\prime}}^{\prime}\left(C_{*}^{\prime}\right) \int_{C_{*}} \exp \left[-\left(a^{\prime}-\epsilon\right)\left(u_{C}(t)\right)^{p^{\prime}}\right] \exp \left[|y| \rho_{C} u_{C}(t)\right]\left|D_{t}^{\gamma}(\phi(t))\right| d t \\
& \leq M^{\prime \prime}\left(C_{*}^{\prime}\right)\|\phi\|_{k_{\alpha}^{\prime}} \int_{C_{*}^{\prime}} \exp \left[-\left(a^{\prime}-\epsilon\right)\left(u_{C}(t)\right)^{p^{\prime}}+|y| \rho_{C} u_{C}(t)\right](1+|t|)^{-n-1} d t \\
& \leq M^{\prime \prime}\left(C_{*}^{\prime}\right)\|\phi\|_{k_{\alpha}^{\prime}} \exp \left[\frac{1}{p}\left(\frac{1}{p^{\prime}\left(a^{\prime}-2 \epsilon\right)}\right){ }^{p / p^{\prime}} \rho_{C}^{p}|y|^{p}\right] \int_{\mathbb{R}^{n}}(1+|t|)^{-n-1} d t
\end{aligned}
$$

where $M^{\prime \prime}\left(C_{*}^{\prime}\right)$ is a constant and $k_{\alpha}^{\prime}$ is a positive integer depending on $\alpha$. Because of (3.3), we can assume that $\epsilon>0$ in (4.22) is fixed such that $\left(a^{\prime}-2 \epsilon\right)>0$. Since (4.22) holds for each $\alpha$ and $\gamma, \beta+\gamma=\alpha$, in (4.19) 
and since $\Phi$ is a bounded set in $\delta$, it follows from the combination of $(4.19),(4.20),(4.21)$, and $(4.22)$ that

$\left\{\left\langle e^{-y t} v_{t}, \phi(t)\right\rangle: \phi \varepsilon \Phi, y \varepsilon O(C),|y| \leq Q\right\}$ is a bounded set in the complex plane for $Q>0$ arbitrary but fixed. Since $\Phi$ was assumed to be an arbitrary bounded set in $\&$, this proves that $\left\{e^{-y t} v_{t}: y \varepsilon O(C),|y| \leq Q\right\}$ is a strongly bounded set in $S^{\prime}$; hence $\left.\left\{\mathrm{F}^{-y t} \mathrm{e}_{t}\right] \varepsilon S^{\prime}: \mathrm{y} \varepsilon 0(\mathrm{C}),|\mathrm{y}| \leq \mathrm{Q}\right\}$ is a strongly bounded set in $\delta^{\prime}$ since the Fourier transform in $\delta^{\prime}$ [2, Chapter 7] is a strongly continuous mapping from $\delta^{\prime}$ onto $\delta^{\prime}$. The proof is complete.

5. ADDITIONS TO THEOREMS 1 AND 2.

Let us now consider Theorem 1. Let $C$ be an open connected cone. Let $f(z) \varepsilon H_{p}(a+\epsilon ; C), p>1$ and $a>0$. By the sufficiency of Theorem 1 we have that the spectral function $v_{t}$ of $f(z)$ has the form (3.2) and

$$
f(z)=\left\langle V_{t}, e^{i z t}\right\rangle, z \varepsilon T^{C}
$$

(Recal1 (1.2).) Further note that $v_{t} \varepsilon 8^{\prime}$ and $\left(e^{-y t} v_{t}\right) \varepsilon \delta^{\prime}$ for all $y \varepsilon O(C)$ as obtained in the proofs of Lemmas 1 and 2 . For any fixed $y \varepsilon C$, $f(x+i y) \varepsilon S^{\prime}$ as a function of $x \in \mathbb{R}^{n}$ because of the growth (3.1) defining the $\mathrm{H}_{\mathrm{p}}(\mathrm{a}+\epsilon ; \mathrm{C})$ spaces. Let $\Psi \varepsilon \&$ and let $\phi \varepsilon \&$ be that unique element of $\delta$ such that $\phi(t)=\mathcal{F}[\Psi(x) ; t] \quad[2$, Chapter 7]. Using (5.1), (3.2), distributional differentiation, a change of order of integration, and differentiation under the integral sign we get

$$
\begin{aligned}
\langle f(z), \Psi(x)\rangle= & \sum_{\alpha}(-1)|\alpha|{ }_{i}|\alpha| \int_{\mathbb{R}^{n}} z^{\alpha} \Psi(x) \int_{\mathbb{R}^{n}} g_{\alpha}(t) e^{i z t} d t d x \\
= & \sum_{\alpha}(-1)|\alpha| \int_{\mathbb{R}^{n}} g_{\alpha}(t)\left(D_{t}^{\alpha} \int_{\mathbb{R}^{n}} \Psi(x) e^{i z t} d x\right) d t .
\end{aligned}
$$

But if $\phi(t)=\mathfrak{F}[\Psi(x) ; t]$ then

$$
\mathrm{e}^{-\mathrm{yt}} \phi(\mathrm{t})=\int_{\mathbb{R}^{\mathrm{n}}} \Psi(\mathrm{x}) \mathrm{e}^{\mathrm{izt}} \cdot \mathrm{dx} .
$$


Putting (5.3) into (5.2) and using the Fourier transform on $\delta^{\prime}$ [2, Chapter 7] we have

$$
\begin{gathered}
\langle f(z), \Psi(x)\rangle=\sum_{\alpha}(-1)^{|\alpha|} \int_{\mathbb{R}^{n}} g_{\alpha}(t)\left(D_{t}^{\alpha}\left(e^{-y t} \phi(t)\right)\right) d t \\
=\left\langle e^{-y t} v_{t}, \phi(t)\right\rangle=\left\langle\widetilde{z}\left[e^{-y t} v_{t}\right], \Psi(x)\right\rangle
\end{gathered}
$$

for all $y=\operatorname{Im}(z) \varepsilon C$ which proves that

$$
f(z)=x\left[e^{-y t} v_{t}\right], z=x+i y \varepsilon T^{C} \text {, }
$$

with this equality holding in $\mathrm{g}^{\prime}$. Thus by combining (5.5) and Lemma 2 we can also conclude in the sufficiency of Theorem 1 that

$\{f(z): y=\operatorname{Im}(z) \varepsilon C,|y| \leq Q\}$ is a strongly bounded set in $\delta^{\prime}$ for $Q>0$ being arbitrary but fixed. Further, by combining (5.5) and Lemma 1 we have obtained a direct proof of the fact that

$$
\lim _{y \rightarrow 0} f(x+1 y)=y[v]
$$

In the strong (and weak) topology of $8^{\prime}$.

In the converse of Theorem 1 vladimirov proves that if $v_{t}$ has the form (3.2) then all derivatives $D_{z}^{\beta}(f(z))$ of the Fourier-Laplace transform $f(z)=\left\langle v_{t}, e^{1 z t}\right\rangle$ of $v_{t}$ belong to the class $H_{p}\left(a \rho_{C}^{p}+\epsilon ; 0(c)\right), c$ being an open connected cone. By the analysis in (5.2), (5.3), and (5.4) we conclude that (5.5) holds in this converse also for $z=x+1 y \in T^{0(C)}$. Then combining this fact with Lemmas 1 and 2 we add the conclusions to the converse of Theorem 1 that $\{f(x): y=\operatorname{Im}(z) \in 0(C),|y| \leq Q\}$ is a strongly bounded set in $\delta^{\prime}$, where $Q>0$ is arbitrary but fixed, and (5.6), with $C$ replaced by $O(C)$, holds in the strong (and weak) topology of $\dot{\delta}^{\prime}$.

We now consider Theorem 2. For the element $f(z) \varepsilon H_{1}(a+\epsilon ; C)$ $\left(\varepsilon \mathrm{H}_{1}\left(\mathrm{a} \rho_{\mathrm{C}} ; 0(\mathrm{C})\right)\right.$ in the converse), a $\geq 0$, and its corresponding spectral function $v_{t} \in 8^{\prime}$ in both the sufficiency and necessity of this theorem, we can 
prove lemmas like Lemmas 1 and 2 . Then using techniques as in our preceding additions to Theorem 1 we have the conclusions in both the sufficiency and necessity of Theorem 2 that

$$
f(z)=\dddot{F}\left[e^{-y t} v_{t}\right], z=x+i y \varepsilon T^{C}\left(\varepsilon T^{0(C)} \text { in the converse }\right),
$$

with this equality holding in $g^{\prime} ;\{f(z): y=\operatorname{Im}(z) \varepsilon C(\varepsilon 0(C)$ in the converse), $|y| \leq Q\}$ is a strongly bounded set in $\mathbf{g}^{\prime}$ for $Q>0$ being arbitrary but fixed; and (5.6) holds in the strong (and weak) topology of $8^{\prime}$ with 0 (C) replacing $\mathrm{C}$ in the converse. The now evident details are left to the interested reader.

Let us also note the generalization of Theorems 1 and 2 given by Vladimirov in [1, section 26.7] concerning functions $f(z) \varepsilon H_{p}(a+\epsilon ; C)$ which are analytic in tubular cones $T^{C}$ where $C$ is an open cone that is the union of a finite number of open connected component cones $C_{k}, k=1,2, \ldots, r$. By our analysis in this paper one can also conclude our strong boundedness property in $\varepsilon^{\prime}$ for the analytic function $f(z) \varepsilon H_{p}\left(a+\epsilon_{;} C\right)$ in $[1, p .247$, Theorem] in each of the connected components $T^{C_{k}}, k=1,2, \ldots, r$, of $T^{C}$ and for the analytic extension function $f(z)$ in the conclusion of this result of vladimirov for $z \in \mathrm{T}^{0(\mathrm{C})}$.

The Theorems 1 and 2 of Vladimirov have recently motivated this author to define more general spaces of analytic functions in tubes than the $\mathrm{H}_{\mathrm{p}}(\mathrm{a} ; \mathrm{C})$ and $H_{p}(a+\epsilon ; C)$ spaces. The associated spectral functions are distributions of exponential growth, a class of distributions which contains the tempered distributions $\mathbf{S}^{\prime}$. Our analysis will appear in [5].

ACKNOWLEDGEMENT. The author expresses his sincere appreciation to the Department of Mathematics of Iowa State University for the opportunity of serving as Visiting Associate Professor during 1978-1979. The author's permanent address is Department of Mathematics, Wake Forest University, Winston-Salem, North Carolina 27109, U.S.A. 


\section{REFERENCES}

1. VLADIMIROV, V. S. Methods of the Theory of Functions of Many Complex Variables, M.I.T. Press, Cambridge, 1966.

2. SCHWARTZ, L. Théorie des Distributions, Hermann, Paris, 1966.

3. VLADIMIROV, V.S. On Functions Holomorphic in Tubular Cones, Izv. Akad. Nauk SSSR Ser. Mat. 27 (1963) 75-100.

4. EDWARDS, R. E. Functional Analysis, Holt, Rinehart and Winston, New York, 1965.

5. CARMICHAEL, Richard D. Analytic Functions Related to the Distributions of Exponential Growth, SIAM J. Math. Anal. (to appear). 


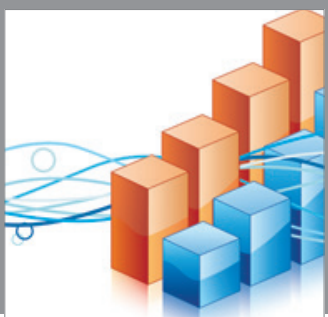

Advances in

Operations Research

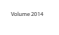

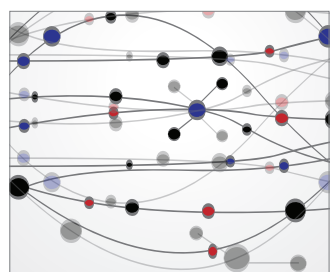

\section{The Scientific} World Journal
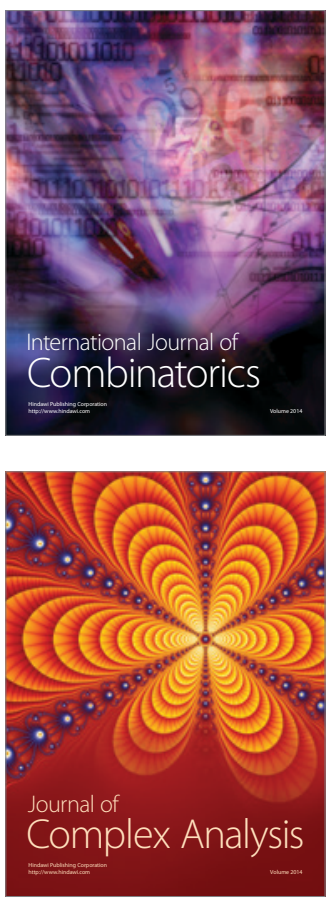

International Journal of

Mathematics and

Mathematical

Sciences
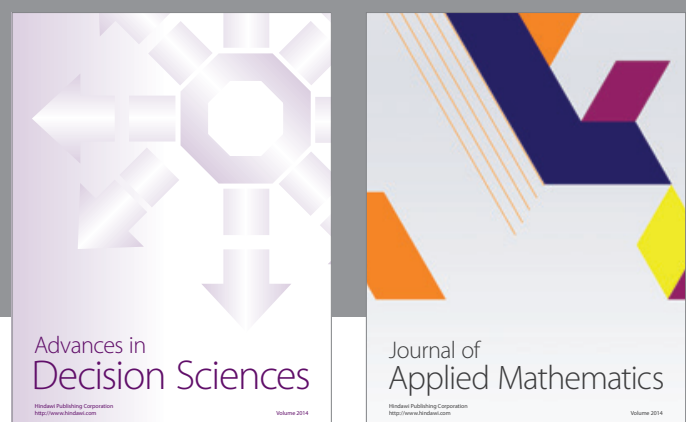

Journal of

Applied Mathematics
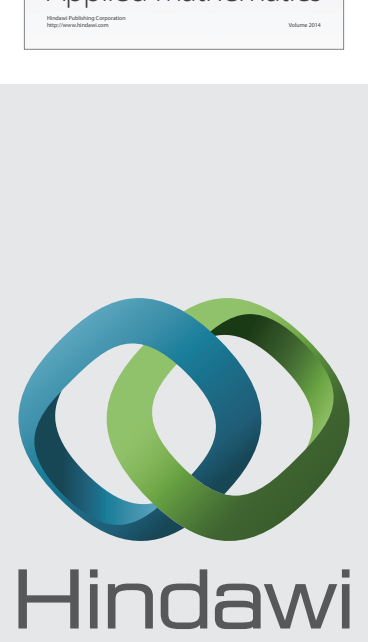

Submit your manuscripts at http://www.hindawi.com
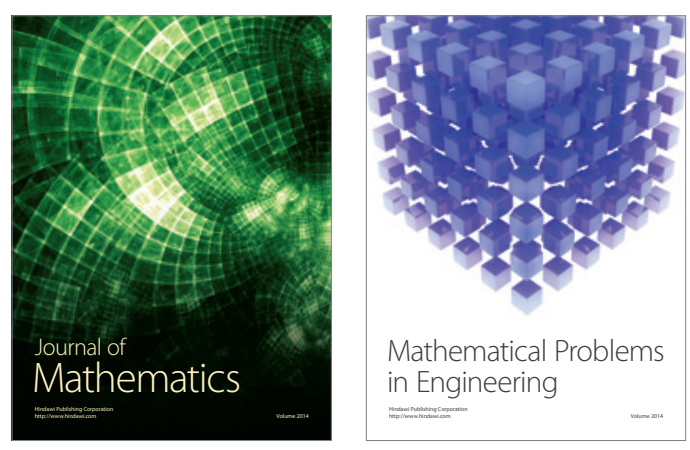

Mathematical Problems in Engineering
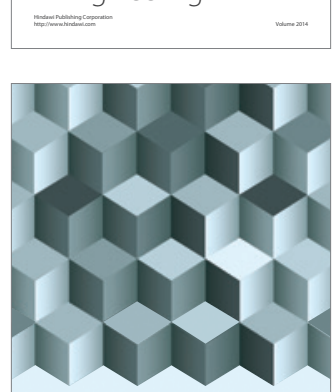

Journal of

Function Spaces
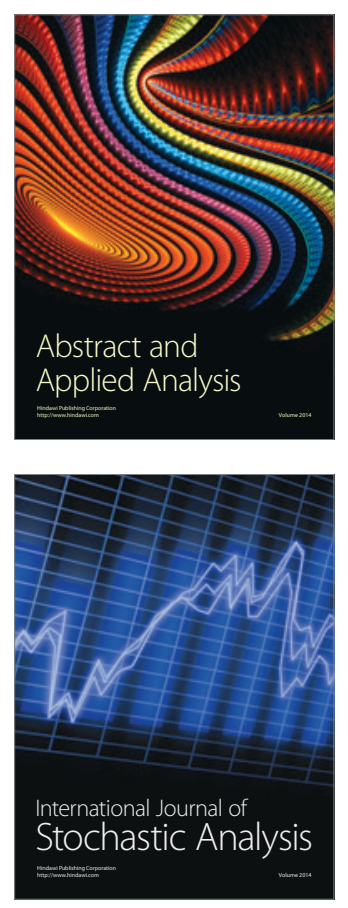

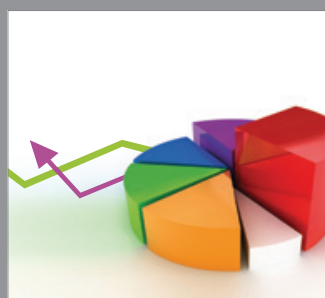

ournal of

Probability and Statistics

Promensencen
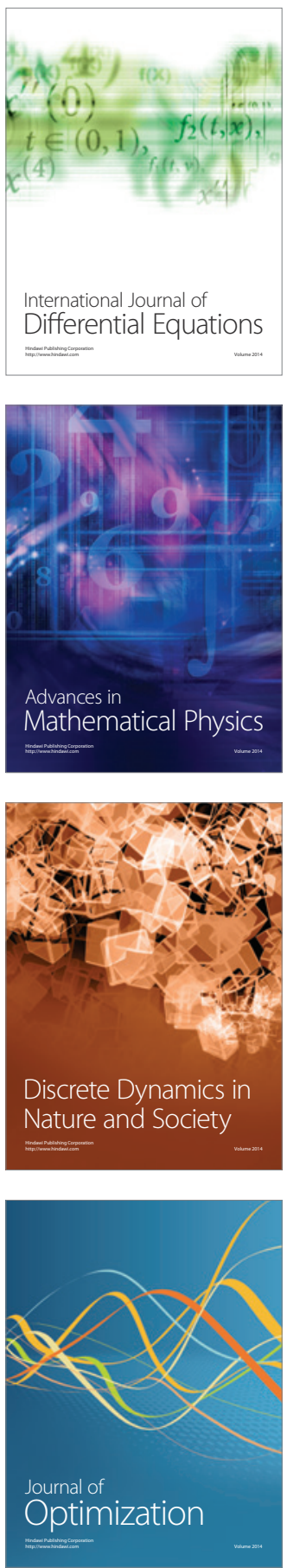